Voix et Images

volxetimages

\title{
De la rhétorique et de la violence
}

\section{Lori Saint-Martin}

Volume 32, numéro 1 (94), automne 2006

Denis Vanier

URI : https://id.erudit.org/iderudit/014712ar

DOI : https://doi.org/10.7202/014712ar

Aller au sommaire du numéro

Éditeur(s)

Université du Québec à Montréal

ISSN

0318-9201 (imprimé)

1705-933X (numérique)

Découvrir la revue

Citer cet article

Saint-Martin, L. (2006). De la rhétorique et de la violence. Voix et Images, 32(1),

152-156. https://doi.org/10.7202/014712ar

Ce document est protégé par la loi sur le droit d'auteur. L'utilisation des services d'Érudit (y compris la reproduction) est assujettie à sa politique d'utilisation que vous pouvez consulter en ligne.

https://apropos.erudit.org/fr/usagers/politique-dutilisation/
Cet article est diffusé et préservé par Érudit.

Érudit est un consortium interuniversitaire sans but lucratif composé de l’Université de Montréal, l’Université Laval et l'Université du Québec à Montréal. Il a pour mission la promotion et la valorisation de la recherche. https://www.erudit.org/fr/ 


\author{
F É M I I S M E S \\ De la rhétorique et de la violence \\ $+++$
}

LORI SAINT-MARTIN

Université du Québec à Montréal

En apparence antinomiques, les deux termes de mon titre ont en commun qu'ils ouvrent des brèches dans les relations entre le masculin et le féminin. La rhétorique au féminin, parce qu'il s'agit d'un moyen de persuader, de justifier une prise de parole longtemps interdite aux femmes, en somme de s'attaquer, en doux et de l'intérieur, aux privilèges discursifs masculins. La violence textuelle au féminin, parce qu'elle pulvérise les stéréotypes du masculin et du féminin en fracassant la représentation au moyen de mille astuces... rhétoriques. Bref, autant les auteures des quatre derniers siècles étudiées dans l'ouvrage collectif dirigé par Annette Hayward ${ }^{1}$ que les protagonistes violentes qu'analyse Paula Ruth Gilbert ${ }^{2}$ ouvrent des brèches transgressives, les unes à coups de plume, les autres à coups de couteau ou de fusil.

Si la visée de la rhétorique consiste surtout à persuader son interlocuteur, les hommes ont été avantagés depuis le début, ainsi que le remarque Diane DesrosiersBonin, non seulement parce que les femmes n'avaient pas le droit d'étudier la rhétorique sous l'Ancien Régime, mais aussi et surtout parce que "la première chose dont [elles] doivent convaincre leur destinataire dans ce contexte est qu'il est légitime pour elles de délaisser la quenouille pour prendre la plume» (87). Tout en s'excusant de leur «rude et mal agencé langaige [sic]» (Jeanne Flore citée par Desrosiers-Bonin, 89), car les femmes, disent-elles, ne peuvent aspirer aux mêmes hauteurs littéraires que les hommes, elles prennent, mine de rien, une certaine place dans l'espace public. Transgression déjà bien grande en soi puisque, comme le rappelle Annette Hayward dans son introduction très utile, puis dans son étude de quelques écrivaines québécoises des débuts, les femmes n'ont pas été autorisées à

1 Annette Hayward (dir.), La rhétorique au féminin, Québec, Nota bene, 2006, 497 p. Le livre est issu d'un colloque international tenu à l'Université Queen's de Kingston. 2 Paula Ruth Gilbert, Violence and the Female Imagination: Quebec's Women Writers Re-Frame Gender in North American Cultures, Montréal/Kingston, McGillQueen's University Press, 2006, 426 p. 
s'exprimer en public et il leur a fallu, pour y parvenir, déployer des stratégies subtiles et indirectes. Quatre siècles plus tard, Hélène Monette, étudiée ici par Lucie Joubert, commente ainsi les questions linguistiques: «Un gars parle, une fille fabule. Un gars s'exprime, une fille rush. Un gars discute, une fille se tait.» (Hélène Monette citée par Joubert, 441) Si le ton a beaucoup changé, l'autodénigrement faisant place à l'ironie et à la satire au féminin, la doxa sociale n'a pas beaucoup avancé.

Rhétorique dans le sens de l'art de l'argumentation, rhétorique comme le déploiement des figures de style et l'ornementation du discours : le sens du terme a évolué au fil des siècles, comme le montre bien Annette Hayward. Entre la longue conférence inaugurale de Catherine Kerbrat-Orecchioni ("Converser au féminin ») et l'amusante nouvelle de Lise Gauvin sur la rhétorique des annonces personnelles («Femme cherche homme»), on trouve vingt études posant chacune à sa manière la question des liens entre sexe-genre et rhétorique.

L'éclatement des époques et des genres littéraires étudiés fait que la question implicite - «Qu'est-ce que la rhétorique au féminin?» - ne trouve pas de réponse directe. Comme aucune étude de l'ouvrage ne compare la rhétorique des femmes à celle des hommes et que presque tous les textes traitent d'une seule créatrice, on a du mal à voir ce qui relève du sexe des auteures et ce qui appartient au style propre de chacune, ou encore ce qui s'explique par des préférences réelles et ce qui tient de la stratégie d'autojustification. Un double exemple montrera que deux Québécoises, à moins de vingt ans d'intervalle, usent de stratégies très différentes. En effet, Claire Martin, qu'étudie avec brio Patricia Smart, écrit de façon directe, voire brutale, affiche une confiance à toute épreuve et insiste sur son autorité narrative afin de rendre crédible sa charge terrible contre son père, les religieuses et la société bienpensante de son époque, alors que Germaine Guèvremont, comme le montre finement David Décarie, déploie de longues et complexes séries de métaphores filées suggérant la sensualité, la liberté, mais aussi la folie. Plus d'une collaboratrice (j'utilise le féminin ici par respect de la majorité, 20 collaborateurs sur 22 étant de sexe féminin) le signale, il y a non pas une mais des rhétoriques au féminin, des stratégies allant de la simplicité censée garantir la véracité du propos ${ }^{3}$ au déploiement de nombreuses figures de style dans un texte dense et complexe. "Rhétorique du bavardage » chez Madame de Sévigné (Agnès Conacher), "rhétorique de la disjonction » chez Julie Papineau (Marilyn Randall), «rhétorique de l'agentivité » chez Geneviève Brisac (Barbara Havercroft), rhétorique comme "processus de l'altérabilité, un potentiel de changement profond du signifiant comme du signifié» chez Nicole Brossard et Élisabeth Vonarburg (Sylvie Bérard), «rhétorique du visuel» chez Madeleine Monette (Claudine Potvin), «rhétorique de l'humain» chez Marguerite Duras (Catherine Dhavernas), «rhétoriques de la véridicité, de l'ironie et du moralisme» chez Claire Martin (Patricia Smart): le sens du terme s'élargit presque à l'infini. On reste sur une impression d'extrême diversité, malgré un certain nombre

$$
+++
$$

3 En effet, au seizième siècle, selon Diane Desrosiers-Bonin (90), certaines femmes adoptent en quelque sorte une rhétorique de la non-rhétorique, un refus de l'ornementation destiné à faire paraître plus vraie leur parole. 
de traits récurrents: présence de l'ironie, de l'humour et de la satire (Lucie Joubert, Susanne Böhmisch), par exemple, grande place faite à la sensualité, voire à la réputation scabreuse de l'auteure, parfois déconsidérée en raison de ses mœurs (Stella Spriet, Christine Klein-Lataud, Élisabeth Zawisza, Jurate Kaminskas), ou encore prédilection pour l'oral et le quotidien qui tire vers le conte (Aron Kibédi Varga, Tamara El-Hoss) ou la lettre (Élisabeth Zawisza, Agnès Conacher, Marilyn Randall). Mais en fait, toutes les attitudes se côtoient ici, de celle de Claire Martin - qui consiste à "écrire "comme un homme" » (335), comme le dit avec ironie Patricia Smart, désireuse de revendiquer, pour les femmes, le droit à tous les registres d'expression - à celle de Geneviève Amyot, étudiée par Louise Dupré, qui sursature l'écriture de marques du féminin (tellement qu'on a peut-être cru à une «écriture de bonne femme», donc peu prestigieuse). Écrire «comme un homme» serait donc plus rentable qu'écrire "comme une femme». Entre ces deux perspectives opposées, de nombreuses voies s'ouvrent à celles qui cherchent leur voix. Impossible à résumer, La rhétorique au féminin impressionne par sa solidité et sa diversité.

«Vous êtes violente; on se tient à distance de la violence, c'est le défaut le plus contraire à votre sexe, qui est complaisant, tendre et doux ${ }^{4}$ ", écrit Denis Diderot à Marie-Madeleine Jodin en 1765. Aujourd'hui comme à l'époque des Lumières, dans l'imagination populaire comme dans les statistiques officielles, la violence a un sexe: elle est masculine. Fermez les yeux, dites « agresseur », "tueur» ou surtout "tueur en série» et voyez les images qui surgissent, conformément à la fois au genre grammatical et aux stéréotypes de genre (homme/femme, activité/passivité, agression/ douceur). Le mot "victime», en revanche, évoque une image féminine, même si, dans la réalité, la majorité des crimes violents ont un homme non seulement pour auteur, mais aussi pour cible.

L'idée persistante selon laquelle la douceur serait inhérente à la nature féminine a pour envers la vision de la femme violente comme un monstre dénaturé: vamp ou vampire, femme fatale, succube, hystérique déchaînée. En raison autant de son caractère exceptionnel que des tabous qu'elle enfreint, la violence des femmes fascine: elle fait peur, elle attire comme une forme de folie.

En littérature comme dans la vraie vie, beaucoup de femmes refoulent cependant leur violence ou la retournent contre elles-mêmes. L'importante étude de Paula Ruth Gilbert traite plutôt des œuvres littéraires, écrites par des femmes, dans lesquelles les personnages féminins s'approprient la violence: sexualité crue et active, violence érotisée, infanticide, meurtres individuels et en série. Cette lecture exhaustive et minutieuse situe le phénomène de la violence textuelle "au féminin » dans le triple contexte des stéréotypes socio-sexuels concernant la violence, abordés

$$
++
$$

4 Cité par Élisabeth Zawisza dans Annette Hayward, op. cit., p. 163. Notons le paradoxe qu'il y a à dire à une femme: "Vous êtes violente», ce qui laisse croire à un trait de caractère inné ou du moins à un comportement spontané, alors que la suite du commentaire insiste sur la douceur naturelle du «beau sexe». 
plus haut, de la nouvelle fascination médiatique pour ce qu'on voit à tort comme une montée en flèche de la violence féminine et de l'américanisation grandissante des cultures canadienne et québécoise, évolution qui irait dans le sens d'une exacerbation de la violence.

L'une des très grandes qualités de ce livre est l'ampleur de la documentation sur laquelle il repose. Paula Gilbert a tout lu, semble-t-il, non seulement en littérature québécoise, mais aussi dans plusieurs domaines - criminologie, sociologie, études féministes et études du genre, théorie littéraire - touchant de près ou de loin son sujet. Son approche multidisciplinaire, nourrie d'études en provenance de l'univers tant anglo-saxon que francophone, lui permet d'aborder de très larges questions liées à l'éthique, au crime, au droit, aux médias, au corps, au désir et à la sexualité, au genre sexuel et textuel, à la représentation au sens large, etc. Scrupuleuse, Paula Gilbert reconnaît avec bonne grâce la contribution de tous les chercheurs et critiques qui ont exploré la question avant elle et cherche à prolonger le dialogue.

Après avoir montré, au moyen d'une longue analyse des statistiques officielles, que le Québec figure parmi les provinces canadiennes les moins violentes et que la criminalité féminine demeure très marginale en Amérique du Nord (le nombre de crimes contre la propriété commis par les femmes augmente, mais pas le nombre d'homicides), Paula Gilbert se lance dans son étude des crimes commis dans les livres. Si elle s'attarde tout particulièrement à cinq auteures - Hélène Rioux, Anne Dandurand et Claire Dé à la fois pour l'interaction du sexe et de la violence et pour la représentation des tueurs et tueuses en série, Suzanne Jacob et Line Chamberland pour l'infanticide -, les nombreuses mentions qu'elle fait d'autres auteures, à défaut de toujours approfondir la compréhension que nous en avons, permet de mesurer l'ampleur du phénomène.

Que signifie cette exploration textuelle de la violence? Selon Paula Gilbert, le refus du statut de victime et l'affirmation de soi par l'action (même s'il s'agit d'une action négative) fracassent les stéréotypes du masculin et du féminin, de manière à nous inviter à repenser à fond la construction sociale des genres. Il s'agit donc d'une étrange - et paradoxale - forme de féminisme, qui consiste à imiter ce que les hommes ont sans doute de pire, soit leur violence. En effet, comment se réjouir de voir des femmes tuer? Si la violence est l'une des caractéristiques les plus troublantes de la masculinité, comment interpréter son essor chez les femmes? Même s'il ne peut s'agir de célébrer ce phénomène, il convient à tout le moins de tenter de le comprendre.

L'extrême ambivalence de ces représentations fascine Paula Gilbert, qui ne tâche jamais d'en diminuer la complexité. Cette ambivalence prend de multiples formes. Par exemple, chez Dandurand et Dé, les femmes s'affirment par l'action, certes, mais souvent par dépit amoureux et par ruse, pour retenir captif ou pour anéantir un homme qu'elles aiment trop. L'idée qu'une femme amoureuse soit prête à tout conforte les stéréotypes. De même, si on dépeint les femmes en succubes, en vampires ou en bêtes de sexe, innove-t-on ou revient-on à des représentations culturelles tristement familières? Et l'inversion des rôles sexuels (l'homme en victime, la femme en bourreau) renouvelle-t-elle réellement les représentations? 
Au plan rhétorique et représentationnel au sens large, l'une des principales stratégies utilisées pour «faire passer » la violence consiste à la déréaliser. À ce titre, Rioux, Dandurand, Dé et de nombreuses autres privilégient l'ironie, l'humour mordant et la parodie, ainsi que la multiplication des niveaux de narration et les stratégies (verbes au conditionnel, modalisateurs semant le doute sur l'action décrite, identités ambiguës), destinées à brouiller la distinction entre le réel et la fiction d'une part, le rêve et le fantasme de l'autre. Ainsi, la violence devient un jeu, une performance, voire une forme de jouissance. Seules exceptions, les infanticides étudiés, qui troublent peut-être encore plus que les autres crimes commis dans les livres, en raison de la vulnérabilité des petites victimes mais aussi du fait qu'ils s'inscrivent dans un cadre représentationnel bien réel, sans le caractère ludique des écrits sur le sexe et la violence. Dans ceux-ci, le recours aux formes brèves, également fréquent, permet la démultiplication des formes d'agression - et de transgression - possibles; cette concentration narrative extrême encourage le fantasme, voire le fantastique, les situations limites et l'idée que la violence, qui reste largement impunie, est sans conséquence. Voici justement l'un des plus grands paradoxes du livre: ces formes que Paula Gilbert situe si patiemment dans le contexte social actuel de l'Amérique du Nord et du Québec contemporains problématisent, voire nient, leurs rapports avec le réel.

À des questions aussi vastes que la représentation de la violence (et la violence de la représentation), la remise en cause des stéréotypes du masculin et du féminin, l'influence de la culture nord-américaine sur les créatrices québécoises et la transformation des relations entre les hommes et les femmes, il est impossible d'apporter des réponses définitives. Paula Gilbert a le mérite de les aborder de front et de les traiter avec méthode et courage. 